\title{
Anomalously slow spin dynamics and short-range correlations in the quantum spin ice systems $\mathrm{Yb}_{2} \mathrm{Ti}_{2} \mathrm{O}_{7}$ and $\mathrm{Yb}_{2} \mathrm{Sn}_{2} \mathrm{O}_{7}$
}

\author{
A. Maisuradze,,${ }^{1,2}$ P. Dalmas de Réotier, ${ }^{3,4}$ A. Yaouanc, ${ }^{3,4}$ A. Forget, ${ }^{5}$ C. Baines, ${ }^{2}$ and P. J. C. King ${ }^{6}$ \\ ${ }^{1}$ Department of Physics, Tbilisi State University, Chavchavadze 3, GE-0128 Tbilisi, Georgia \\ ${ }^{2}$ Laboratory for Muon-Spin Spectroscopy, Paul Scherrer Institute, CH-5232 Villigen-PSI, Switzerland \\ ${ }^{3}$ Université Grenoble Alpes, INAC-SPSMS, F-38000 Grenoble, France \\ ${ }^{4}$ CEA, INAC-SPSMS, F-38000 Grenoble, France \\ ${ }^{5}$ CEA, Centre de Saclay, DSM/IRAMIS/Service de Physique de l'Etat Condensé, F-91191 Gif-sur-Yvette, France \\ ${ }^{6}$ ISIS Facility, Rutherford Appleton Laboratory, Chilton, Didcot, OX11 0QX, United Kingdom
}

(Received 12 June 2015; published 14 September 2015)

\begin{abstract}
We report a positive muon spin relaxation and rotation $(\mu \mathrm{SR})$ study of the quantum spin ice materials $\mathrm{Yb}_{2} \mathrm{Ti}_{2} \mathrm{O}_{7}$ and $\mathrm{Yb}_{2} \mathrm{Sn}_{2} \mathrm{O}_{7}$ focusing on the low field response. In agreement with earlier reports, data recorded in small longitudinal fields evidence anomalously slow spin dynamics in the microsecond range below the temperature $T_{\mathrm{c}}$ at which the specific heat displays an intense peak, namely $T_{\mathrm{c}}=0.24 \mathrm{~K}$ and $0.15 \mathrm{~K}$, respectively, for the two systems. We found that slow dynamics extends above $T_{\mathrm{c}}$ up to at least $0.7 \mathrm{~K}$ for both compounds. The conventional dynamical Gaussian Kubo-Toyabe model describes the $\mu$ SR spectra recorded above $T_{\mathrm{c}}$. At lower temperatures a published analytical extension of the Gaussian Kubo-Toyabe model provides a good description, consistent with the existence of short-range magnetic correlations. While the physical response of the two systems is qualitatively the same, $\mathrm{Yb}_{2} \mathrm{Ti}_{2} \mathrm{O}_{7}$ exhibits a much larger local magnetic susceptibility than $\mathrm{Yb}_{2} \mathrm{Sn}_{2} \mathrm{O}_{7}$ below $T_{\mathrm{c}}$. Considering previously reported ac susceptibility, neutron scattering, and $\mu$ SR results, we suggest the existence of anomalously slow spin dynamics to be a common physical property of pyrochlore magnetic materials. The possibility of molecular spin substructures to be associated to the slow dynamics and therefore the short-range correlations is mentioned. The slow spin dynamics observed under field does not exclude the presence of much faster dynamics detected in extremely low or zero field.
\end{abstract}

DOI: 10.1103/PhysRevB.92.094424

PACS number(s): 75.40.-s, 75.10.Jm, 75.10.Kt, 76.75.+i

\section{INTRODUCTION}

The pyrochlore insulator compounds $R_{2} M_{2} \mathrm{O}_{7}$, where $R$ is a rare earth ion and $M$ a nonmagnetic element, have attracted considerable attention. The most remarkable discovery has been the spin-ice ground state of $\mathrm{Ho}_{2} \mathrm{Ti}_{2} \mathrm{O}_{7}$ in 1997 [1]. This ground state is now known for the emergence of a latticebased Coulomb phase characterized by dipolar correlations and therefore pinch-point scattering patterns [2]. Its excitations are emergent magnetic monopoles [3-5].

This first success has led to systematic studies of other members of the $R_{2} M_{2} \mathrm{O}_{7}$ family [6]. However, apparently conflicting results have generated confusion. Only recently clarifying experimental data and thorough analyses have appeared. For example, low temperature magnetization measurements for $\mathrm{Tb}_{2} \mathrm{Ti}_{2} \mathrm{O}_{7}$ barely support the existence of a magnetization plateau expected for a spin-ice-like compound, at least down to $0.02 \mathrm{~K}[7,8]$. A first physical explanation of the persistent spin dynamics at low temperature as observed by the positive muon spectroscopy ( $\mu \mathrm{SR})$ spinlattice relaxation rate has been proposed [9]. The dynamics would arise from low energy unidimensional excitations. They would be supported by spin loops originally discussed in Refs. [10-12].

$\mathrm{Yb}_{2} \mathrm{Ti}_{2} \mathrm{O}_{7}$ stands alone. The originally proposed low temperature spin liquid state, derived from powder sample measurements $[13,14]$, has been challenged ever since, with apparently no agreement reached on its basic physical properties, such as the absence or existence of long-range magnetic correlations at low temperature as probed by neutron diffraction [13,15-17]. Remarkably, these correlations have recently been firmly established for a powder sample of the sibling compound $\mathrm{Yb}_{2} \mathrm{Sn}_{2} \mathrm{O}_{7}[18,19]$.

One of the most mysterious physical properties of magnetic pyrochlore compounds is the existence of anomalously slow spin dynamics. Here, a thorough study of the local magnetic fields and spin dynamics of $\mathrm{Yb}_{2} \mathrm{Ti}_{2} \mathrm{O}_{7}$ and $\mathrm{Yb}_{2} \mathrm{Sn}_{2} \mathrm{O}_{7}$ as probed by $\mu \mathrm{SR}$ spectroscopy under field is reported. Anomalously slow fluctuations of the $\mathrm{Yb}^{3+}$ magnetic moments are detected below and above the temperature $T_{\mathrm{c}}$ at which specific heat displays a pronounced anomaly. Their effect on the measured spectra is fully described. Short-range correlations are detected below $T_{\mathrm{c}}$ for both compounds. $\mathrm{Yb}_{2} \mathrm{Ti}_{2} \mathrm{O}_{7}$ is found to be characterized by a much larger local magnetic susceptibility below $T_{\mathrm{c}}$ than $\mathrm{Yb}_{2} \mathrm{Sn}_{2} \mathrm{O}_{7}$.

The organization of this paper is as follows. Section II introduces the experimental method and explains the analysis of $\mu \mathrm{SR}$ spectra. In Secs. III and IV the $\mathrm{Yb}_{2} \mathrm{Ti}_{2} \mathrm{O}_{7}$ and $\mathrm{Yb}_{2} \mathrm{Sn}_{2} \mathrm{O}_{7}$ experimental spectra and their analysis are respectively presented. In the following section (Sec. V) a discussion of the present data and previously reported extremely low-field results is provided. Conclusions are gathered in Sec. VI.

\section{EXPERIMENTAL AND DATA ANALYSIS}

The measurements were performed on powder samples already used for different experimental investigations $[13,14,18,20-22]$. Both of them are characterized by a well marked change in the longitudinal $\mu \mathrm{SR}$ signal at $T_{\mathrm{c}}$. For $\mathrm{Yb}_{2} \mathrm{Ti}_{2} \mathrm{O}_{7}$ this is in agreement with the results of Ref. [23], but in contrast to Ref. [24] for which a clear $\mu \mathrm{SR}$ signature of $T_{\mathrm{c}}$ 
only appears in the frequency shift. The magnetic transitions at $T_{\mathrm{c}}$ are first order with $T_{\mathrm{c}} \simeq 0.24$ and $0.15 \mathrm{~K}$ for $\mathrm{Yb}_{2} \mathrm{Ti}_{2} \mathrm{O}_{7}$ and $\mathrm{Yb}_{2} \mathrm{Sn}_{2} \mathrm{O}_{7}$, respectively. This was first shown in Refs. [13,18], respectively. Later on, the first order nature of the transition for $\mathrm{Yb}_{2} \mathrm{Ti}_{2} \mathrm{O}_{7}$ was further characterized by detailed magnetization measurements [25]. Hence the magnetic history of a compound is expected to influence results of measurements in its ordered state. When presenting our spectra for $T<T_{\mathrm{c}}$ we shall explicitly describe the experimental conditions under which they were recorded. Surprisingly enough, for both compounds no spontaneous $\mu \mathrm{SR}$ magnetic field has been observed below $T_{\mathrm{c}}$, even for a powder sample of $\mathrm{Yb}_{2} \mathrm{Sn}_{2} \mathrm{O}_{7}$ for which magnetic Bragg reflections have been detected as mentioned in the Introduction.

The $\mu \mathrm{SR}$ measurements were performed at the MuSR spectrometer of the ISIS facility (Rutherford Appleton Laboratory) and the LTF spectrometer of the Swiss Muon Source ( $\mathrm{S} \mu \mathrm{S}$, Paul Scherrer Institute).

The measurements were mostly done with the longitudinal geometry for which the external field $\mathbf{B}_{\text {ext }}$ is set along the initial muon beam polarization $\mathbf{S}_{\mu}$ [26]. In fact, it occurs that $\mathbf{B}_{\text {ext }}$ and $\mathbf{S}_{\mu}$ are not strictly parallel due to the presence of a separator in the beamlines. This is particularly true at LTF [27]. Hence, while the longitudinal polarization function derives from the counts detected in the detectors placed parallel and antiparallel to $\mathbf{B}_{\text {ext }}$, an oscillating signal is observed in the detectors set perpendicular to $\mathbf{B}_{\text {ext }}$. Although its amplitude is small it contains valuable information which we have used in this study. The results obtained in this way were confirmed by recording a few spectra in the usual transverse geometry, i.e., with $\mathbf{S}_{\mu}$ oriented perpendicular to $\mathbf{B}_{\text {ext }}$ [28].

We now present the framework for the data analysis. The polarization function probing a static magnetic field distribution with field component distributions $D_{\mathrm{c}}\left(B^{\alpha}\right)$ identical for the three Cartesian directions $\alpha=X, Y$, or $Z$ is derived from the Larmor equation. In the longitudinal geometry, it is expressed as

$$
\begin{aligned}
P_{Z}^{\text {stat }}(t)= & \frac{1}{\mathcal{Z}} \iiint_{-\infty}^{\infty}\left\{\left(\frac{B^{Z}}{B}\right)^{2}+\left[1-\left(\frac{B^{Z}}{B}\right)^{2}\right] \cos \left(\omega_{\mu} t\right)\right\} \\
& \times D_{\mathrm{c}}\left(B^{X}\right) D_{\mathrm{c}}\left(B^{Y}\right) D_{\mathrm{c}}\left(\frac{B^{Z}-B_{\text {long }}}{\mathcal{Z}}\right) d B^{X} d B^{Y} d B^{Z},
\end{aligned}
$$

with $B^{2}=\left(B^{X}\right)^{2}+\left(B^{Y}\right)^{2}+\left(B^{Z}\right)^{2}, \quad \omega_{\mu}=\gamma_{\mu} B$ where $\gamma_{\mu}$ is the muon gyromagnetic ratio $\left(\gamma_{\mu}=2 \pi \times 135.53 \times\right.$ $\left.10^{6} \mathrm{rad} \mathrm{s}^{-1} \mathrm{~T}^{-1}\right)$, and $B_{\text {long }}$ is the longitudinal field along the $Z$ axis which may not be necessarily equal to $B_{\text {ext }}$. The parameter $\mathcal{Z}$ describes a possible uniaxial anisotropy in the $Z$ direction. Unless $\mathcal{Z}$ is specified, we shall assume $\mathcal{Z}=1$, i.e., no anisotropy.

In fact the field at the muon sites has often a dynamical character. The muon polarization function $P_{Z}(t)$ for a magnetic field fluctuating at rate $v_{\mathrm{c}}$ is computed using the strong collision model [26,29]. It satisfies the following integral equation:

$$
\begin{aligned}
P_{Z}(t)= & P_{Z}^{\mathrm{stat}}(t) \exp \left(-v_{\mathrm{c}} t\right) \\
& +v_{\mathrm{c}} \int_{0}^{t} P_{Z}\left(t-t^{\prime}\right) P_{Z}^{\text {stat }}\left(t^{\prime}\right) \exp \left(-v_{\mathrm{c}} t^{\prime}\right) d t^{\prime} .
\end{aligned}
$$

To proceed further an expression for $D_{\mathrm{c}}\left(B^{\alpha}\right)$ is needed. A conventional choice is a Gaussian function. Understanding $P_{Z}(t)$ measured for $\mathrm{Yb}_{2} \mathrm{Ti}_{2} \mathrm{O}_{7}$ below $T_{\mathrm{c}}$ required the following simple extension of the Gaussian shape [22]:

$$
D_{\mathrm{c}}(x \delta)=\frac{1}{N \delta} \exp \left[-\frac{1}{2} x^{2}-\frac{1}{3}\left(\eta_{3} x\right)^{3}-\frac{1}{4}\left(\eta_{4} x\right)^{4}\right] .
$$

Here, $\delta$ sets the magnetic field scale and $N$ is the normalization constant. In zero field and when $\eta_{3}=\eta_{4}=0$, i.e., for a Gaussian field component distribution, $P_{Z}^{\text {stat }}(t)$ is given by the famous Kubo-Toyabe formula [30]. Under field and still in the Gaussian limit and with $\mathcal{Z}=1$, the threedimensional integral in Eq. (1) reduces to a one-dimensional integral [26]. In zero field only symmetrized distributions are probed [31]. In principle, in longitudinal field, $\mu \mathrm{SR}$ can resolve asymmetric distributions. However, concerning the data presented in this paper, no clear deviation from symmetric distributions was found in a first analysis [32]. Therefore, the distributions entering Eq. (1) have always been symmetrized distributions $D_{\mathrm{c}}^{\mathrm{sym}}\left(B^{\alpha}\right)$ defined as

$$
D_{\mathrm{c}}^{\mathrm{sym}}\left(B^{\alpha}\right)=\frac{1}{2}\left[D_{c}\left(B^{\alpha}\right)+D_{c}\left(-B^{\alpha}\right)\right] .
$$

Once $D_{\mathrm{c}}^{\mathrm{sym}}\left(B^{\alpha}\right)$ is determined, the standard deviation $\Delta_{\mathrm{LF}}$ of the distribution can be computed numerically according to

$$
\Delta_{\mathrm{LF}}^{2}=\int_{-\infty}^{\infty} b^{2} D_{\mathrm{c}}^{\mathrm{sym}}(b) d b,
$$

since the average of $D_{\mathrm{c}}^{\mathrm{sym}}(b)$ is zero. If $\eta_{3}=\eta_{4}=0, \Delta_{\mathrm{LF}}=\delta$.

Equation (3) is an ansatz which assumes the field component distribution to deviate only slightly from the Gaussian shape. It can account for short-range spin-correlation effects [22]. The presence of short-range correlations is already known to transform the Kubo-Toyabe function into a polarization function with a shallower minimum [33]. It is natural to apply Eq. (3) for the description of longitudinal field (LF) $\mu \mathrm{SR}$ asymmetry spectra recorded in a paramagnetic state. It can even be used below $T_{\mathrm{c}}$ when a spontaneous field is not detected, e.g., because of its dynamical nature [34].

LF spectra will be analyzed with the two-component model

$$
A^{\mathrm{LF}}(t)=A_{0}^{\mathrm{LF}}\left[\left(1-F_{\mathrm{bg}}\right) P_{Z}(t)+F_{\mathrm{bg}}\right],
$$

where $A_{0}^{\mathrm{LF}}$ is the initial $\mu \mathrm{SR}$ asymmetry, and $F_{\mathrm{bg}}$ the fraction of muons stopped outside of the sample, for which the relaxation is negligible.

Except for some spectra recorded in the ordered state of $\mathrm{Yb}_{2} \mathrm{Ti}_{2} \mathrm{O}_{7}$, asymmetry time spectra corresponding to the transverse field (TF) geometry will be described with a weighted sum of two Gaussian damped oscillations:

$$
\begin{aligned}
& A^{\mathrm{TF}}(t) \\
& =A_{0}^{\mathrm{TF}}\left[\left(1-F_{2}\right) \exp \left(-\frac{\gamma_{\mu}^{2} \Delta_{\mathrm{TF}, 1}^{2} t^{2}}{2}\right) \cos \left(\gamma_{\mu} B_{1} t+\phi\right)\right. \\
& \left.\quad+F_{2} \exp \left(-\frac{\gamma_{\mu}^{2} \Delta_{\mathrm{TF}, 2}^{2} t^{2}}{2}\right) \cos \left(\gamma_{\mu} B_{2} t+\phi\right)\right] .
\end{aligned}
$$

Here, $\phi$ is an experimental phase parameter and $A_{0}^{\mathrm{TF}}$ the initial asymmetry in the transverse geometry. For the vast majority of measurements for which LF and TF spectra were 

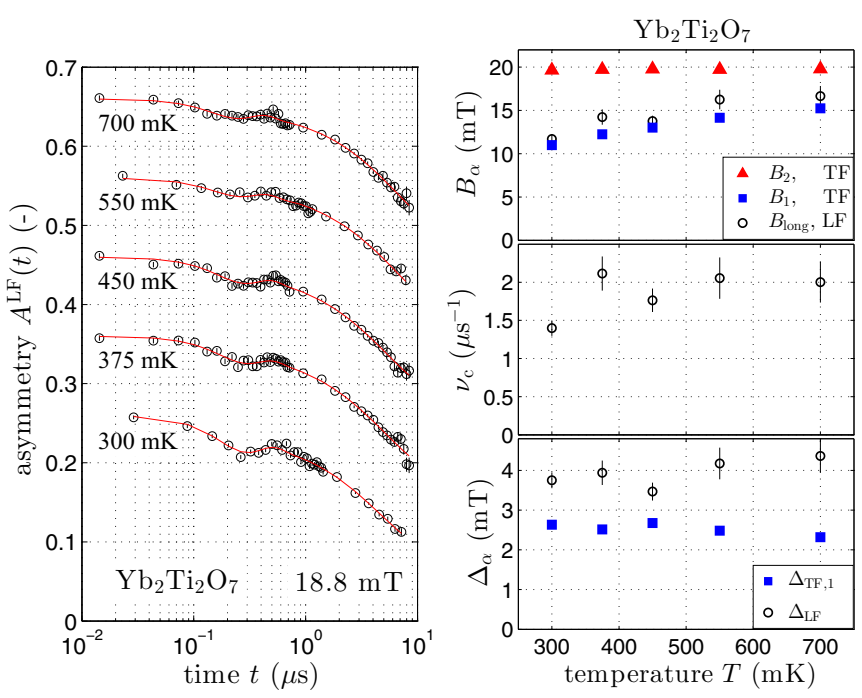

FIG. 1. (Color online) $\mu \mathrm{SR}$ asymmetry spectra recorded at LTF

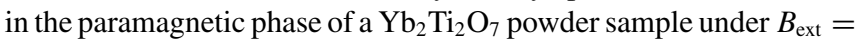
$18.8 \mathrm{mT}$. (Left) Temperature scan of the longitudinal asymmetry. Circles are experimental data while solid lines represent fits as explained in the main text. The spectra for consecutive temperatures are vertically shifted by 0.1 for better visualization. A logarithmic time scale is used. (Right) Thermal dependence of fitting parameters extracted for spectra recorded in the longitudinal and transverse geometries.

simultaneously recorded at $\mathrm{LTF}, A_{0}^{\mathrm{TF}} \simeq 0.06$. The relation $\left(A_{0}^{\mathrm{LF}}\right)^{2}+\left(A_{0}^{\mathrm{TF}}\right)^{2}=0.27^{2}$ holds since the total asymmetry is a characteristic of the spectrometer. The mean fields $B_{1}, B_{2}$, and the standard deviations $\Delta_{\mathrm{TF}, 1}$ and $\Delta_{\mathrm{TF}, 2}$ refer to the first and second components, respectively. We find $\Delta_{\mathrm{TF}, 2} \ll \Delta_{\mathrm{TF}, 1}$, $F_{2}=F_{\text {bg }}$ within experimental uncertainties, and $B_{2} \simeq B_{\text {ext }}$. These results are consistent with the attribution of the second component to the background, i.e., to muons missing the sample. Its narrow signal can serve as a precise measurement of $B_{\text {ext }}$. For the first component we resorted to a simple function rather than trying to take into account a possible deviation from the Gaussian field component distribution [22]. This is justified since, while we shall find deviations from the conventional description for the longitudinal asymmetries at low temperature, the simple expression in Eq. (7) provides a proper description of the measured transverse-field asymmetries.

\section{EXPERIMENTAL RESULTS: $\mathbf{Y b}_{\mathbf{2}} \mathbf{T i}_{2} \mathbf{O}_{7}$}

We shall first consider spectra recorded in the paramagnetic phase, i.e., above $T_{\mathrm{c}} \simeq 0.24 \mathrm{~K}$. In a second step data taken below $T_{\mathrm{c}}$ will be presented.

\section{A. Paramagnetic state}

We first show in Fig. 1 asymmetry spectra recorded with a longitudinal field $B_{\text {ext }}=18.8 \mathrm{mT}$ at different temperatures $T>T_{\text {c }}$. Remarkably, the asymmetry does not decay monotonically, but displays a well defined local minimum, at about $0.25 \mu \mathrm{s}$ at low temperature. The structure is less pronounced as the sample is warmed, but is still discernible at $700 \mathrm{mK}$, pointing to a relatively slow spin dynamics in the paramagnetic state [26]. The spectra were analyzed with Eqs. (1)-(6). The conventional dynamical Gaussian Kubo-Toyabe model was found to provide a good description, i.e., $\eta_{3}=\eta_{4}=0$.

Transverse geometry spectra were analyzed with Eq. (7) and an example of such analysis is shown in the left panel of Fig. 2. In the right panel the Fourier transform (FT) amplitude of the spectrum is displayed. Only two peaks are resolved, in contrast to Ref. [24] where three peaks - one broad and two narrow-are reported for a powder sample, both above and below $T_{\mathrm{c}}$. This will be further discussed at the end of Sec. III B.

The thermal dependences of the parameters extracted from the LF and TF analyses are presented in the three panels on the right of Fig. 1. As already noticed, $B_{2}$ is a good measure of $B_{\text {ext }}$. One can see that both $B_{1}$ and $B_{\text {long }}$ are closely related and are substantially smaller than $B_{2} \simeq B_{\text {ext }}$. These important results will be discussed in Sec. V A. On increasing temperature this reduction is attenuated. The fluctuation rate of the spin dynamics is found to be $v_{\mathrm{c}} \simeq 2 \mu \mathrm{s}^{-1}$ confirming the anomalously slow spin dynamics already inferred from visual examination of the spectra. We find $\Delta_{\mathrm{LF}}$ and $\Delta_{\mathrm{TF}, 1}$ to be
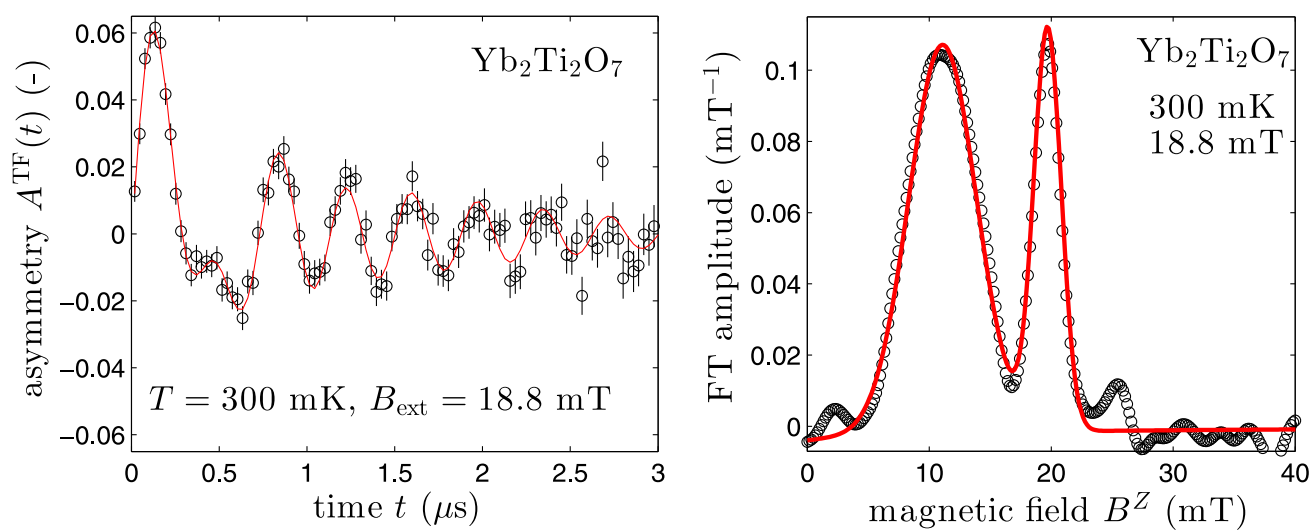

FIG. 2. (Color online) (Left) Example of a transverse-field asymmetry spectrum measured at $300 \mathrm{mK}$ under $B_{\text {ext }}=18.8 \mathrm{mT}$ for a powder sample of $\mathrm{Yb}_{2} \mathrm{Ti}_{2} \mathrm{O}_{7}$. The spectrum was recorded at LTF with the spectrometer in the longitudinal geometry. This explains the small value for the asymmetry. Black circles display the spectrum while the red curve results from a fit to the data with Eq. (7) as explained in the main text. (Right) The corresponding Fourier transform amplitude of the asymmetry data and fitting curve. 

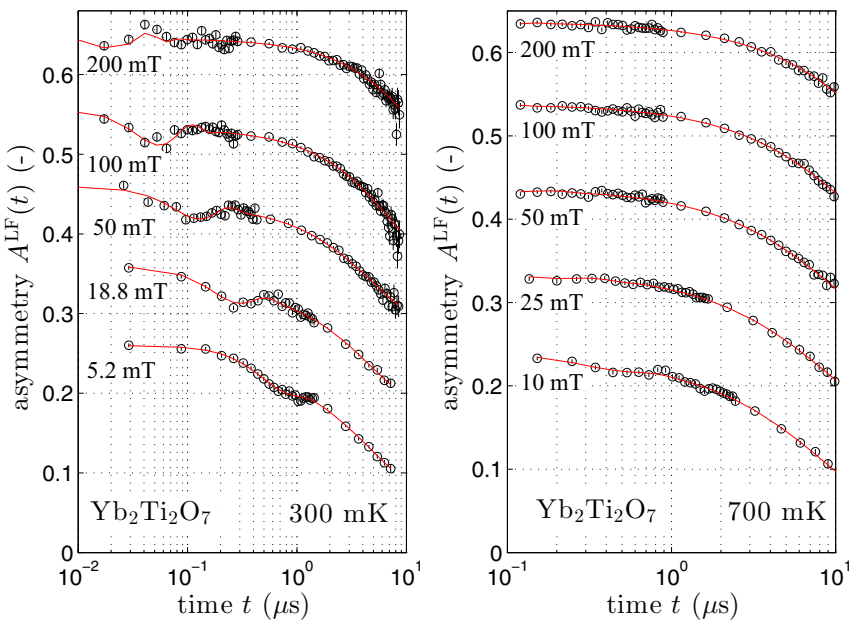

FIG. 3. (Color online) Field dependence of asymmetry spectra recorded in the paramagnetic phase of a $\mathrm{Yb}_{2} \mathrm{Ti}_{2} \mathrm{O}_{7}$ powder sample in the longitudinal geometry. The data are represented by black circles and the corresponding fitting curves as solid lines. The fits are explained in the main text. The spectra were recorded at LTF for $T=300 \mathrm{mK}$ (left) and at MuSR for $T=700 \mathrm{mK}$ (right). The data for consecutive $B_{\text {ext }}$ values (indicated nearby each spectrum) are shifted by 0.1 unit for better visualization.

roughly temperature independent. In contrast to expectation, these parameters are quite different. However, we note that taking $\mathcal{Z}=0.8$, the fit of the spectra are of similar quality and within uncertainties we still get the same results for $B_{\alpha}$ and $v_{\mathrm{c}}$, but now $\Delta_{\mathrm{LF}}$ and $\Delta_{\mathrm{TF}, 1}$ are approximately equal. The anisotropy of the field component distribution that this result suggests could be a field-induced effect. The important point is that $B_{1}$ and $B_{\text {long }}$ remains nearly equal.

In order to confirm the unusually slow spin dynamics we recorded data for a set of longitudinal fields at 300, 450, 550 , and $700 \mathrm{mK}$, i.e., above $T_{\mathrm{c}}$. Examples of spectra and fitting curves are presented in Fig. 3. Again the dynamical Gaussian Kubo-Toyabe model gives a nice description of the spectra. Field-induced oscillations are clearly observed in a broad field range, supporting the existence of slow fluctuations. The fit parameters are plotted in Fig. 4 as a function of $B_{\text {ext }}$ for temperatures up to $550 \mathrm{mK}$. The oscillations amplitude being somewhat reduced when the temperature is increased, some of the fit parameters are indeed poorly defined at higher temperatures. The reduction of the field probed by the muons is confirmed; see the plot of $B_{\alpha}$ versus $B_{\text {ext }}$. The parameters $\Delta_{\mathrm{LF}}$ and $\Delta_{\mathrm{TF}, 1}$ characterizing the standard deviation of the Gaussian field distribution in $\mathrm{LF}$ and $\mathrm{TF}$ geometries, respectively, increase roughly linearly with $B_{\text {ext }}$.

\section{B. Ordered magnetic state}

In a previous work it was shown that understanding a spectrum recorded at $200 \mathrm{mK}$, i.e., below $T_{\mathrm{c}}$, and at low field for $\mathrm{Yb}_{2} \mathrm{Ti}_{2} \mathrm{O}_{7}$ required an extension of the conventional Gaussian distribution model [22]. This was confirmed with a numerical method based on the maximum entropy principle and the reverse Monte Carlo algorithm [31]. In Fig. 5(a) we display spectra recorded for different longitudinal fields at 70 $\mathrm{mK}$, i.e., below $T_{\mathrm{c}}$, at LTF. Spectra taken with the same experi-

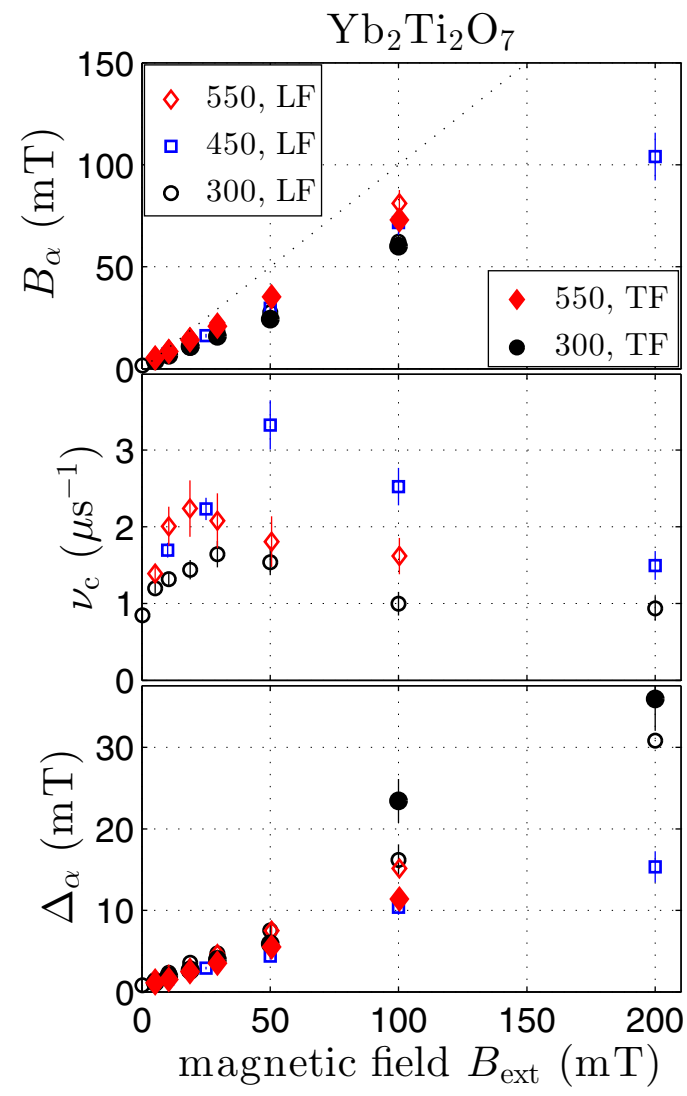

FIG. 4. (Color online) External magnetic field dependence of physical parameters extracted from the analysis of field scans performed in the paramagnetic phase of a $\mathrm{Yb}_{2} \mathrm{Ti}_{2} \mathrm{O}_{7}$ powder sample. We first plot $B_{\text {long }}$ and $B_{1}$ (collectively denoted as $B_{\alpha}$ ), then $v_{\mathrm{c}}$, and finally $\Delta_{\mathrm{LF}}$ and $\Delta_{\mathrm{TF}, 1}$ (collectively denoted as $\Delta_{\alpha}$ ). Most of the data have been derived from LF spectra (empty symbols), with few results obtained with the TF (full symbols) geometry at 300 and $550 \mathrm{mK}$. The plotting symbols correspond to different temperatures (given in units of millikelvin) and experimental geometries as indicated in the insets.

mental conditions at MuSR were published elsewhere [20]. Here we profit from the better time resolution available at LTF. The sample was zero-field cooled. The data were subsequently recorded for increasing values of the field. Weak oscillations are observed, with an amplitude which is again reduced at high field. The asymmetry spectra were analyzed with Eqs. (1)-(6). The separate analysis of the spectra led to parameters $\eta_{3}$ and $\eta_{4}$ independent of the field, within uncertainty. Therefore, in a second step, the spectra at different fields were simultaneously fit with the same set of parameters $\eta_{3}$ and $\eta_{4}$. The fits shown in Fig. 5(a) are excellent. We found $\eta_{3}=0.73$ (2) and $\eta_{4}=0.46(2)$. This is consistent with the previous analysis of a spectrum recorded at $200 \mathrm{mK}$ under $2 \mathrm{mT}$ [22]. The two parameters $\eta_{3}$ and $\eta_{4}$ are not negligible. This means that $D_{\mathrm{c}}^{\mathrm{sym}}\left(B^{\alpha}\right)$ displayed in Fig. 5(e) deviates substantially from the conventional Gaussian shape as the comparison shows. From the values of $\eta_{3}$ and $\eta_{4}$ we compute the field distribution standard deviation $\Delta_{\mathrm{LF}}=1.70(9) \delta$. Note that the parameters $\eta_{3}$ and $\eta_{4}$ are strongly correlated. The inferred $D_{\mathrm{c}}^{\mathrm{sym}}\left(B^{\alpha}\right)$ is characterized by relatively pronounced tails. The fitting parameters $B_{\text {long }}, v_{\mathrm{c}}$, and $\Delta_{\mathrm{LF}}$ are presented 

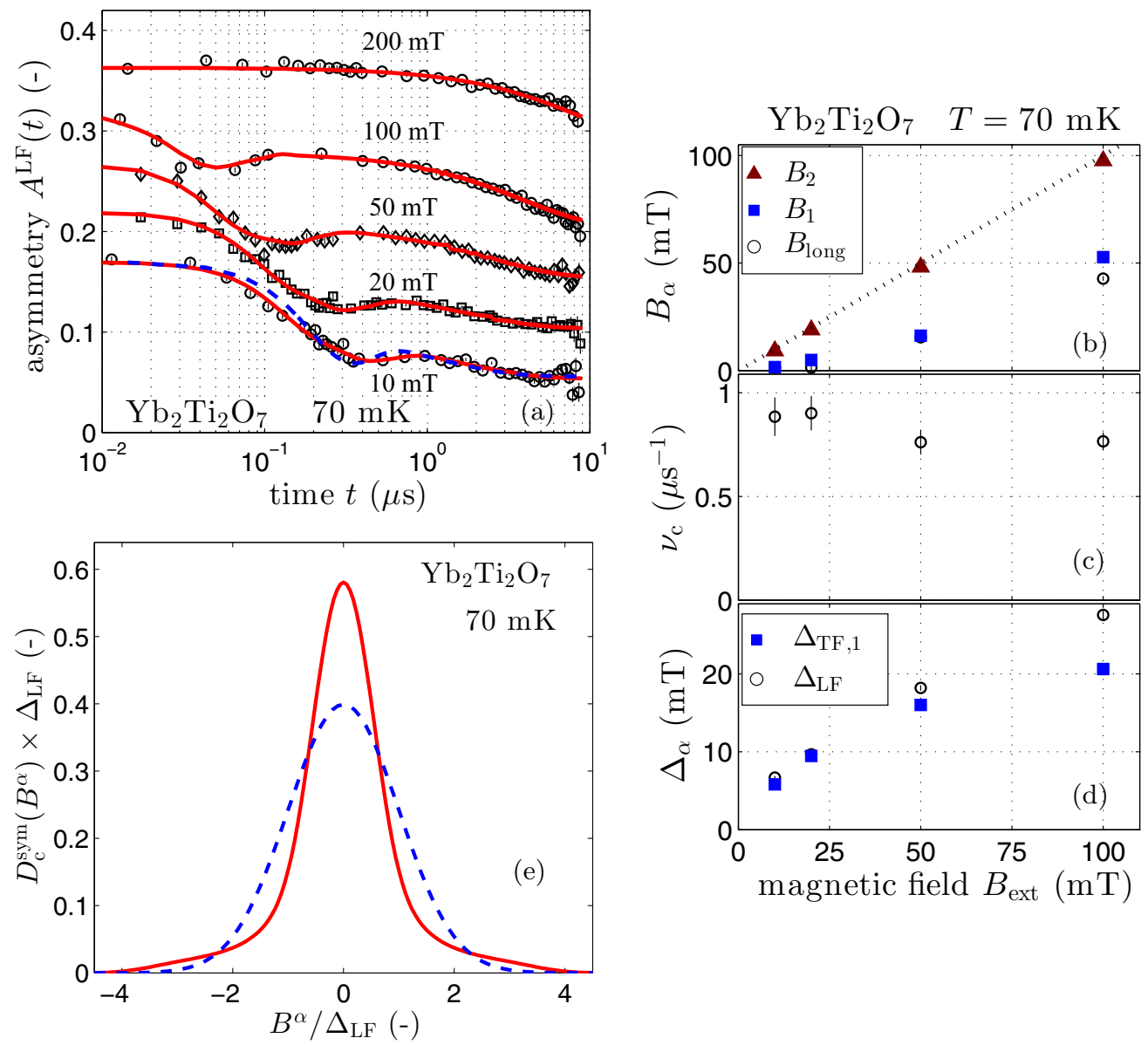

FIG. 5. (Color online) Data recorded for a powder sample of $\mathrm{Yb}_{2} \mathrm{Ti}_{2} \mathrm{O}_{7}$ at $T=70 \mathrm{mK}$ with the longitudinal geometry. (a) Measured asymmetries with increasing $B_{\text {ext }}$ indicated nearby each spectrum. The spectra are vertically shifted by 0.05 for better visualization. The solid lines result from analytical fits as explained in the main text. For comparison, the blue dashed line represents the dynamical Gaussian Kubo-Toyabe fit of the asymmetry measured in a field of $10 \mathrm{mT}$. (b),(c),(d) Empty circles show the field dependence of three parameters describing the asymmetry spectra: the field $B_{\text {long }}$, the fluctuation rate $v_{\mathrm{c}}$, and the standard deviation $\Delta_{\mathrm{LF}}=1.70 \delta$ for $\eta_{3}=0.7335$ and $\eta_{4}=0.4558$. Full symbols represent the field dependence of $B_{1}, B_{2}$, and $\Delta_{\mathrm{TF}, 1}$ obtained from the TF spectra (not shown). The dotted line in panel (b) corresponds to the curve $B_{\text {long }}=B_{\text {ext }}$. (e) Field component distribution in reduced field scale. The solid line results from the analysis with the extension of the Gaussian model. The dashed line is for the Gaussian field distribution corresponding to the dynamical Gaussian Kubo-Toyabe fit in panel (a).

in Figs. 5(b)-5(d). We find $B_{\text {long }} \ll B_{\text {ext }}$ with $B_{\text {long }}$ roughly proportional to $B_{\text {ext }}$, as in the paramagnetic state but with a much smaller coefficient of proportionality. The fluctuation rate $v_{\mathrm{c}} \lesssim 1 \mu \mathrm{s}^{-1}$ is smaller than in the paramagnetic state but still appreciable. The standard deviation $\Delta_{\mathrm{LF}}$ increases quite linearly with field, in agreement with the behavior in the paramagnetic state. $\Delta_{\mathrm{TF}, 1}$ is slightly different from $\Delta_{\mathrm{LF}}$ at high field. As for the paramagnetic state, this difference becomes negligible if a small field-induced anisotropy is introduced. This is a side effect which can be neglected, as already mentioned when the paramagnetic data were discussed.

The strong reduction of $B_{\text {long }}$ relative to $B_{\text {ext }}$ is remarkable. As expected, and supporting our data analysis, $B_{1} \simeq B_{\text {long }}$. We expand on the meaning of this reduction in Sec. V A.

In order to resolve the discrepancy between our results and those reported in Ref. [24], we performed additional measurements at the LTF spectrometer in TF configuration. This allows us to simultaneously record LF and TF spectra with a large $A_{0}^{\mathrm{TF}}=0.21$, and a smaller $A_{0}^{\mathrm{LF}}=0.17$. The ex- perimental conditions, namely measurements in field cooling protocol with $B_{\text {ext }}=50 \mathrm{mT}$ and at $T=50 \mathrm{mK}$, are as in Ref. [24]. The result is shown in Fig. 6. Qualitatively our Fourier spectrum is consistent with that reported in Ref. [24]. Three peaks are visible. This is in contrast to the spectrum displayed in Fig. 2 for which only two peaks are present. Regarding this latter spectrum, $B_{\text {ext }}$ is smaller and the data concern the paramagnetic instead of the ordered state. While it is natural to assign the broad peak around $20 \mathrm{mT}$ in Fig. 6 to the sample, we believe that the other two arise from muons stopped outside the sample. Indeed, the relative contributions of the peaks at 46 and $49 \mathrm{mT}$ to the Fourier spectrum are $14(1) \%$ and $12(1) \%$, respectively. Assigning only the peak at $49 \mathrm{mT}$ to the background leads to an unreasonably small background value for the LTF spectrometer. Most probably, the stray field and the geometry of the sample and sample holder are at the origin of the two peaks on the right of the field scale. The narrow peak should correspond to the part of the sample-holder located far from the sample while a slightly 

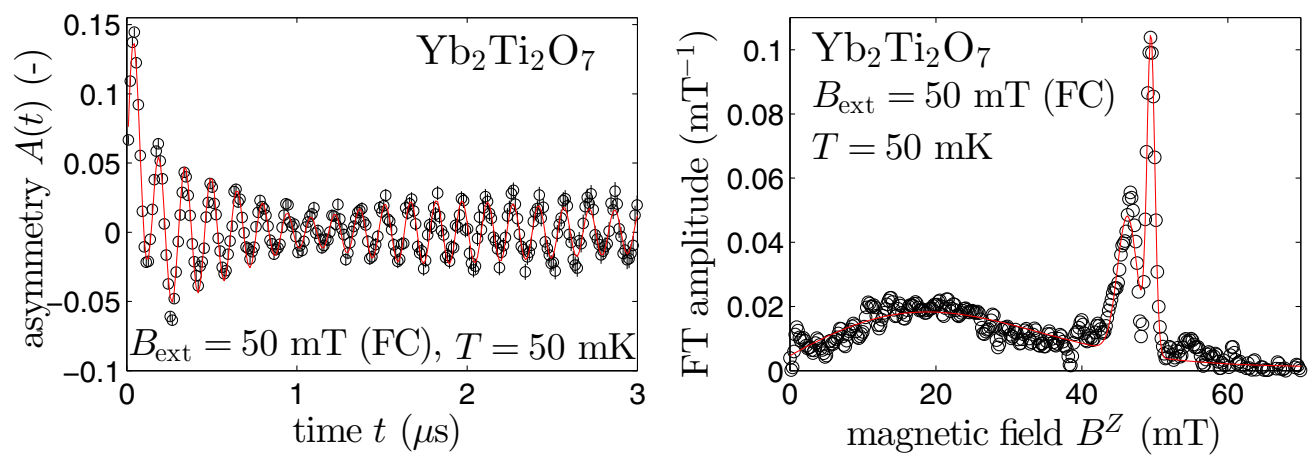

FIG. 6. (Color online) (Left) Example of a transverse-field asymmetry spectrum measured at $50 \mathrm{mK}$ under $B_{\text {ext }}=50 \mathrm{mT}$ in field cooling condition for a powder sample of $\mathrm{Yb}_{2} \mathrm{Ti}_{2} \mathrm{O}_{7}$. The spectrum was recorded at LTF with the spectrometer in the transverse geometry. Black circles display the spectrum while the red curve results from a fit with a model similar to Eq. (7) but with three Gaussian oscillating components. (Right) The corresponding Fourier transform amplitude of the asymmetry data and fitting curve. The broad signal stems from the sample while two narrow peaks at $B^{Z} \simeq 46 \mathrm{mT}$ and $B^{Z} \simeq 49 \mathrm{mT}$ originate from muons stopped outside the sample.

broader peak originates from the sample-holder part in the immediate neighborhood to the sample.

In any case, the agreement demonstrated in Fig. 5(b) between the mean fields $B_{1}$, measured directly from TF spectra, and $B_{\text {long }}$, extracted from the analysis of LF spectra, is a strong support for the methodology of our TF and LF data analysis.

\section{EXPERIMENTAL RESULTS: $\mathrm{Yb}_{2} \mathrm{Sn}_{2} \mathrm{O}_{7}$}

Here we present results of the analysis for $\mathrm{Yb}_{2} \mathrm{Sn}_{2} \mathrm{O}_{7}$ in the same way as was done for $\mathrm{Yb}_{2} \mathrm{Ti}_{2} \mathrm{O}_{7}$. We first focus on the paramagnetic state.

\section{A. Paramagnetic state}

In Fig. 7 we display results obtained in a LF of $B_{\text {ext }}=18.8$ $\mathrm{mT}$ above $T_{\mathrm{c}}=0.15 \mathrm{~K}$. Also in this compound one can clearly see oscillations in the asymmetry spectra associated with $B_{\text {ext }}$, which directly point to a slow spin dynamics. The dynamical Gaussian Kubo-Toyabe model, i.e., Eqs. (1)-(6) with $\eta_{3}=\eta_{4}=0$ provides a good account of the spectra. The local mean field, i.e., $B_{\text {long }}$, in this compound is again reduced. The strength of the reduction is weaker than in $\mathrm{Yb}_{2} \mathrm{Ti}_{2} \mathrm{O}_{7}$ and it also decreases with increasing temperature. The difference between $B_{1}$ and $B_{\text {long }}$ is small and may not be really significant. The spin fluctuation rate $v_{\mathrm{c}}$ gradually increases with temperature and reaches a maximal value of 2.0 (2) $\mu \mathrm{s}^{-1}$ at $700 \mathrm{mK}$. As expected, the magnitudes of $\Delta_{\mathrm{TF}, 1}$ and $\Delta_{\mathrm{LF}}$ are almost equal. This is in contrast to $\mathrm{Yb}_{2} \mathrm{Ti}_{2} \mathrm{O}_{7}$.

In order to clarify the origin of the slow spin dynamics in the paramagnetic state additional measurements at 250, 420, and $550 \mathrm{mK}$ were performed for different fields. In Fig. 8 we show examples at 250 and $550 \mathrm{mK}$, together with the fitting curves depicted as solid lines. The oscillations due to $B_{\text {ext }}$ are present in the whole field range of the measurements. Again, a Gaussian field component distribution is sufficient for the description of the spectra. In Fig. 9 the corresponding fitting parameters are presented as a function of field. The field dependence of the three parameters measured for the stannate and the titanate in their paramagnetic states is rather similar.

\section{B. Ordered magnetic state}

Spectra were recorded in the magnetically ordered state at $14 \mathrm{mK}$. They were presented in Ref. [18]. The sample was first zero-field cooled and then $\mathbf{B}_{\text {ext }}$ was applied with the following sequence: 5, 3.4, 9.7, 19, 50, and $100 \mathrm{mT}$. The LF and TF asymmetry spectra are well described with Eqs. (1)-(6) and Eq. (7), respectively. As for $\mathrm{Yb}_{2} \mathrm{Ti}_{2} \mathrm{O}_{7}$ the parameters $\eta_{3}$ and $\eta_{4}$ are appreciable and field independent within the statistical uncertainties. Therefore, we performed a global fit of the LF spectra with common $\eta_{3}$ and $\eta_{4}$ in order to determine the field distribution. The experimental LF time asymmetries together with the fitting curves are presented in Fig. 10(a). The analysis results in $\eta_{3}=0.67$ (4) and $\eta_{4}=0.40(3)$, i.e., only slightly
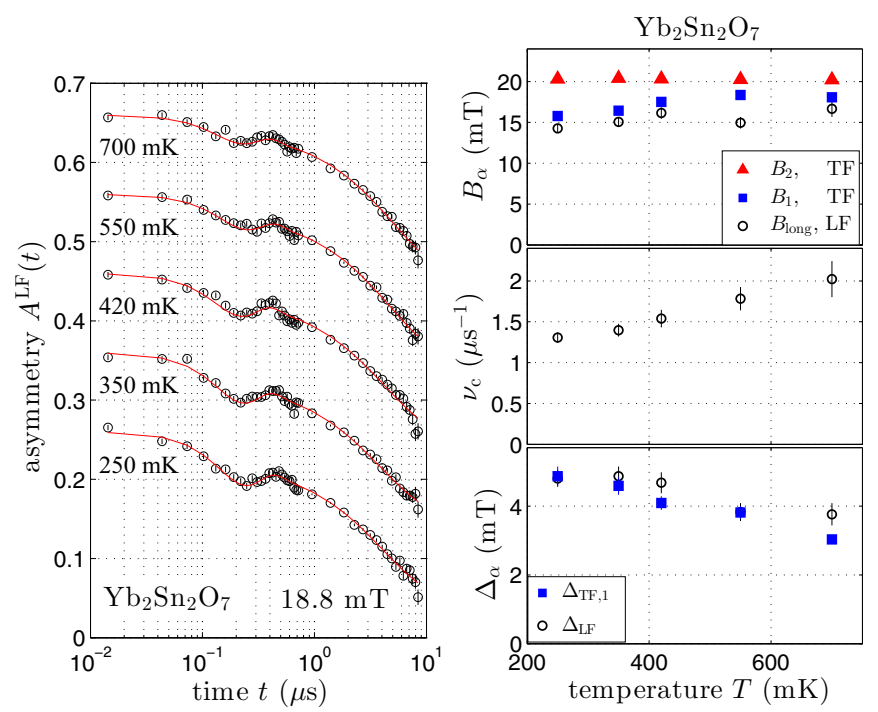

FIG. 7. (Color online) Results obtained in the paramagnetic phase of a powder sample of $\mathrm{Yb}_{2} \mathrm{Sn}_{2} \mathrm{O}_{7}$ under $B_{\text {ext }}=18.8 \mathrm{mT}$. (Left) Examples of asymmetry spectra obtained at LTF in the longitudinal geometry. Circles are experimental data while solid lines represent fits as explained in the main text. The temperature is given nearby each of the spectra which are vertically shifted by 0.1 units for better visualization. (Right) Parameters extracted from a fit to Eqs. (1)-(6) for LF data and Eq. (7) for TF data. 

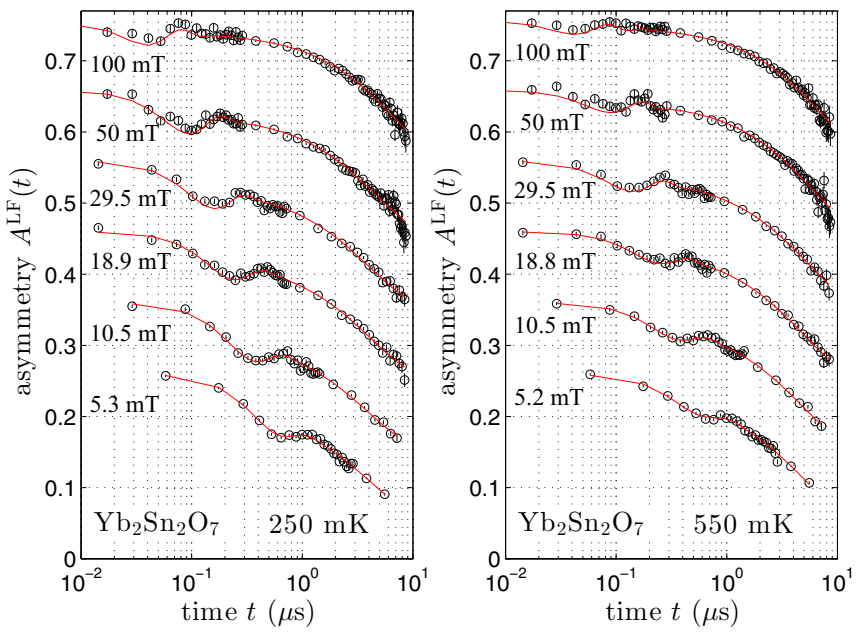

FIG. 8. (Color online) Examples of field scan measurements of asymmetry spectra performed in the paramagnetic phase of a powder sample of $\mathrm{Yb}_{2} \mathrm{Sn}_{2} \mathrm{O}_{7}$ (black circles) and corresponding fitting curves obtained with Eqs. (1)-(6). The spectra were recorded in the longitudinal geometry at the LTF spectrometer for $T=250 \mathrm{mK}$ (left) and $T=550 \mathrm{mK}$ (right). The $B_{\text {ext }}$ value is given nearby the spectra which are shifted by 0.1 unit relative to each other for better visualization. It is remarkable that oscillations due to $B_{\mathrm{ext}}$ are clearly observed pointing to a low frequency spin dynamics above $T_{\mathrm{c}}$ in $\mathrm{Yb}_{2} \mathrm{Sn}_{2} \mathrm{O}_{7}$.

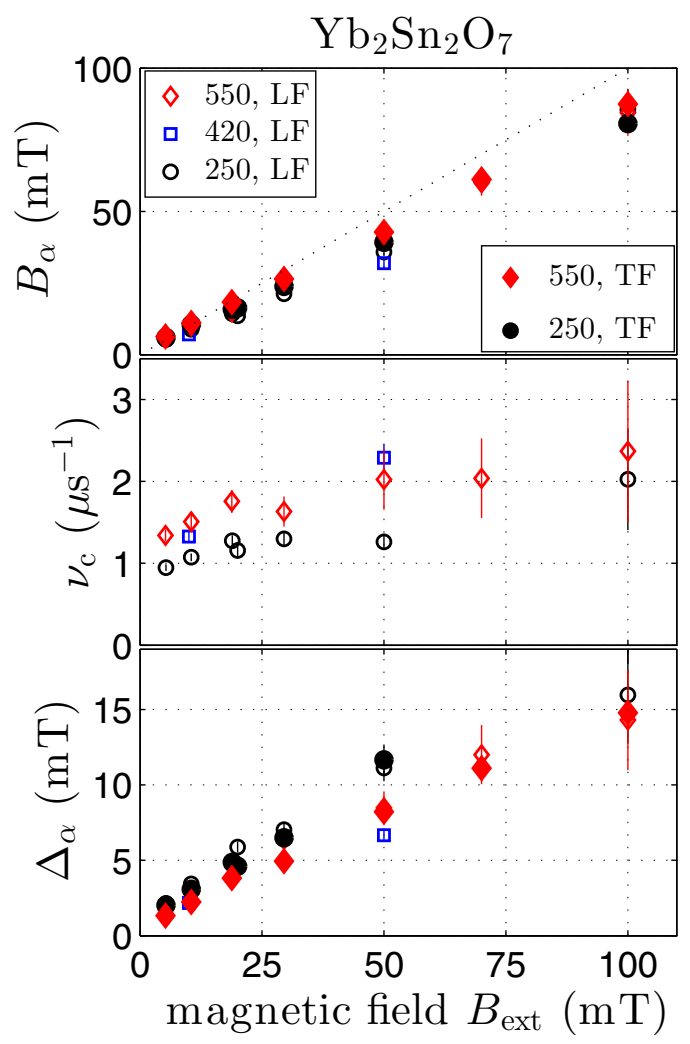

FIG. 9. (Color online) Same caption as for Fig. 4. Here the physical parameters concern the paramagnetic phase of $\mathrm{Yb}_{2} \mathrm{Sn}_{2} \mathrm{O}_{7}$.
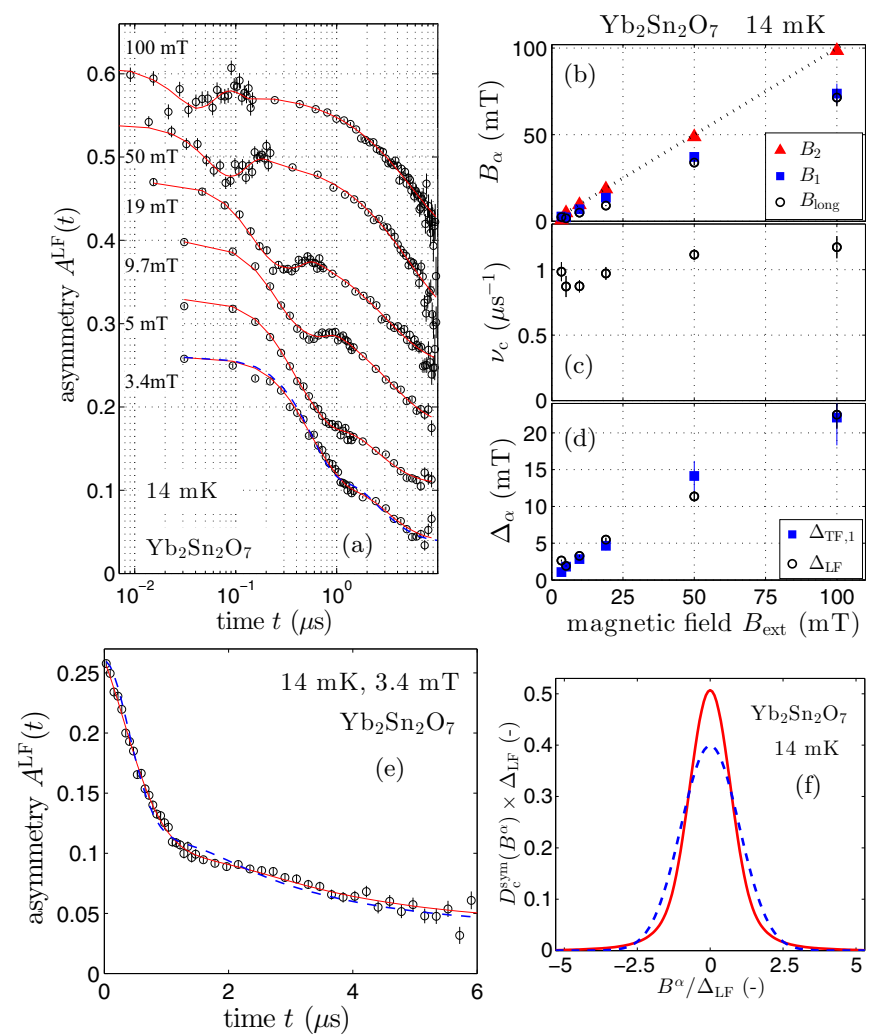

FIG. 10. (Color online) Data recorded at the LTF spectrometer for a powder sample of $\mathrm{Yb}_{2} \mathrm{Sn}_{2} \mathrm{O}_{7}$ at $T=14 \mathrm{mK}$ with the longitudinal geometry. (a) Field dependence of LF time spectra (circles) and fits (solid lines) as described in the main text. The spectra are vertically shifted by 0.07 for better visualization. (b),(c),(d) Corresponding fitting parameters as a function of $B_{\text {ext }}$. The field component standard deviation is $\Delta_{\mathrm{LF}}=1.38 \delta$ for $\eta_{3}=0.670$ and $\eta_{4}=0.400$. The $B_{\mathrm{ext}}$ dependence of the parameters deduced from the TF spectra is also displayed. (e) LF time spectrum (circles) at $3.4 \mathrm{mT}$ plotted with a linear time scale and fits with the extension of the Gaussian model in red solid line and the conventional Gaussian Kubo-Toyabe model in blue dashed line. (f) Field component distributions in normalized field units. The solid line shows the distribution obtained from a global fit using the extension of the Gaussian model, and the dashed line the Gaussian distribution corresponding to the Kubo-Toyabe fit in panel (e), also in blue dashed line.

different from the values measured for $\mathrm{Yb}_{2} \mathrm{Ti}_{2} \mathrm{O}_{7}$. From these values we get $\Delta_{\mathrm{LF}}=1.38$ (9) $\delta$. In Figs. 10(b)-10(d) we show the field dependence of the other LF parameters, together with the TF parameters. A linear dependence of the field standard deviations is also present in the ordered state. The fluctuation rate $v_{\mathrm{c}}$ is slightly reduced and exhibits a weaker field dependence than in the paramagnetic state. The fields $B_{\text {long }}$ and $B_{1}$ are equal within experimental uncertainties. They are reduced relative to $B_{\text {ext }}$, but not as strongly as in the case of $\mathrm{Yb}_{2} \mathrm{Ti}_{2} \mathrm{O}_{7}$; see Fig. 5. In Fig. 10(e) we compare the results of the fits with the Gaussian model and with its extension. The latter solution provides a better description. The field component distribution obtained from this analysis is shown in Fig. 10(f) as a solid line. As for $\mathrm{Yb}_{2} \mathrm{Ti}_{2} \mathrm{O}_{7}$, a significant deviation from the Gaussian shape displayed as a dashed line is evident: pronounced tails are present. 


\section{DISCUSSION}

We shall first consider the conclusions reached here from the analysis of spectra recorded under longitudinal fields. Then we shall discuss their compatibility with those previously derived from extremely low field measurements.

\section{A. Measurements under relatively large fields}

Let us first summarize the results of the analysis of the $\mu \mathrm{SR}$ spectra for the two compounds discussed in this report, i.e., $\mathrm{Yb}_{2} \mathrm{Ti}_{2} \mathrm{O}_{7}$ and $\mathrm{Yb}_{2} \mathrm{Sn}_{2} \mathrm{O}_{7}$.

The following features emerge. From the highest temperature investigated, i.e., $700 \mathrm{mK}$, down to far below the temperature $T_{\mathrm{c}}$ at which a sharp peak in the specific heat is detected, the fluctuation rate of the $\mathrm{Yb}^{3+}$ magnetic moments is equal to $\nu_{\mathrm{c}} \approx 1 \mu \mathrm{s}^{-1}$, independent of the field and temperature within a factor 2 . Hence the spin dynamics is anomalously slow. The component field distribution measured under longitudinal field is Gaussian above $T_{\mathrm{c}}$ and somewhat deviates from it below $T_{\mathrm{c}}$. Its standard deviation increases linearly with the field for the two compounds, both below and above $T_{\mathrm{c}}$. When normalized to the width, the distribution is field independent in the probed field range. Finally, a reduction of the mean field at the muon site is measured for the two compounds. Within experimental uncertainties, it is similar in longitudinal and transverse field measurements. It is particularly strong for $\mathrm{Yb}_{2} \mathrm{Ti}_{2} \mathrm{O}_{7}$ at $T<T_{\mathrm{c}}$. Remarkably, it is approximately linear in field, both below and above $T_{\mathrm{c}}$. The ratio $\left(B_{\alpha}-\right.$ $\left.B_{\text {ext }}\right) / B_{\text {ext }}$ can therefore be considered as a local magnetic susceptibility.

As the magnetic phase transitions are first order, the procedure followed to record spectra below $T_{\mathrm{c}}$ has an influence on the results of the measurements. We recall that the asymmetry spectra analyzed in this paper were taken after cooling the samples in zero field, except for the data of Fig. 6.

We now discuss the meaning of our results. We first note that the existence of slow spin dynamics in magnetic pyrochlore compounds is well documented. It was reported in 2002 for $\mathrm{Yb}_{2} \mathrm{Ti}_{2} \mathrm{O}_{7}$ from the analysis of $\mu \mathrm{SR}$ and Mössbauer measurements [13]. Soon after, it was realized that a wide spectrum of fluctuation rates exists in this compound [15]. Later on, slow spin dynamics was reported for the pyrochlore $\mathrm{Tb}_{2} \mathrm{Ti}_{2} \mathrm{O}_{7}$ using ac susceptibility measurements [35]. Slow spin dynamics with a wide range of fluctuation rates of the rare-earth magnetic moments in geometrically frustrated magnetic compounds is in fact one of their characteristics. As an additional example, we mention the garnet compound $\mathrm{Gd}_{3} \mathrm{Ga}_{5} \mathrm{O}_{12}$ with three-dimensional hyperkagomé magnetic lattice for which neutron-scattering studies reveal a remarkable wide range of energies from milli- to picoelectronvolt [36]. The present study puts on firm ground the existence of slow dynamics for $\mathrm{Yb}_{2} \mathrm{Ti}_{2} \mathrm{O}_{7}$ and $\mathrm{Yb}_{2} \mathrm{Sn}_{2} \mathrm{O}_{7}$ as seen by $\mu \mathrm{SR}$.

As to the physical mechanism at the origin of the slow dynamics, we note that persistent spin dynamics at low temperature, i.e., below $\simeq 1 \mathrm{~K}$, as observed by $\mu \mathrm{SR}$ spinlattice relaxation in pyrochlore compounds, has recently been proposed to arise from unidimensional excitations that would be supported by spin loops [9]. These one-dimensional objects in three-dimensional crystal structures such as the pyrochlore structure have been studied early on $[10,12]$. Their existence provides a natural explanation for slow dynamics since it means that spin loops, i.e., a large number of spins in contrast to a conventional single spin, are involved in the dynamics. This picture may extend above $\simeq 1 \mathrm{~K}$, the interaction between spin loops being recognized. This is in qualitative agreement with the observation of slow spin dynamics deep into the paramagnetic state of $\mathrm{Nd}_{2} \mathrm{Sn}_{2} \mathrm{O}_{7}$ [37]. Interestingly, classical simulations of spin dynamics for the antiferromagnetic Heisenberg model on the kagomé lattice is consistent with the loop picture; see Ref. [38], and references therein.

The field component distribution extracted from the measurements below $T_{\mathrm{c}}$ deviates from the Gaussian shape for both compounds, pointing out the existence of short-range correlations. These correlations are absent in the ordered magnetic state of a conventional compound. They do exist for $\mathrm{Er}_{2} \mathrm{Ti}_{2} \mathrm{O}_{7}$ [39]. The increase of the field standard deviation with $B_{\text {ext }}$ suggests that the field induces some disorder. The mechanism at play is not clear. It could be that the field induces a canting of the magnetic moments. Surprisingly, we have not found any effect of magnetic short-range correlations on the field component distribution in the paramagnetic state. Yet such correlations, signalled by a broad hump in the specific heat centered at about 3 and $2 \mathrm{~K}$ for $\mathrm{Yb}_{2} \mathrm{Ti}_{2} \mathrm{O}_{7}$ and $\mathrm{Yb}_{2} \mathrm{Sn}_{2} \mathrm{O}_{7}$, respectively, do exist $[18,21,40,41]$ and have even been observed by neutron scattering $[42,43]$ in a crystal of $\mathrm{Yb}_{2} \mathrm{Ti}_{2} \mathrm{O}_{7}$ whose specific heat does nonetheless display no sharp peak at $T_{\mathrm{c}}$ [21]. The detection of these correlations by $\mu \mathrm{SR}$ needs to be further investigated.

Let us now discuss the origin for the reduction of the mean field at the muon site. We have the key result that the same reduction is measured for LF and TF asymmetries. This suggests that in fact we are dealing with a frequency shift effect and not an effect of intermittency of the field at the muon site [44]. This interpretation is supported by the results published for $\mathrm{Tb}_{2} \mathrm{Ti}_{2} \mathrm{O}_{7}$ [45]. In this system, below $10 \mathrm{~K}$, the relative frequency shift varied between -0.2 and -0.7 . In $\mathrm{Yb}_{2} \mathrm{Ti}_{2} \mathrm{O}_{7}$ [Fig. 5(b)] it is approximately -0.6 and for $\mathrm{Yb}_{2} \mathrm{Sn}_{2} \mathrm{O}_{7}$ [Fig. 10(b)] it is near -0.2 . In conclusion, there is no exotic physics in the measured field reduction. It just occurs that the frequency shift is negative. If the muon site localization were known, it would probably be possible to understand this feature. This has recently been done for $\mathrm{MnSi}$ [46]. The much larger reduction below $T_{\mathrm{c}}$ for $\mathrm{Yb}_{2} \mathrm{Ti}_{2} \mathrm{O}_{7}$ relative to $\mathrm{Yb}_{2} \mathrm{Sn}_{2} \mathrm{O}_{7}$ is just the signature of a much larger local magnetic susceptibility for the former compound.

\section{B. Compatibility with previously published extremely low field measurements}

The asymmetry $A^{\mathrm{LF}}(t)$ and therefore the polarization function $P_{Z}(t)$ in the paramagnetic state does not decay monotonically at intermediate $B_{\text {ext }}$ values for $\mathrm{Yb}_{2} \mathrm{Ti}_{2} \mathrm{O}_{7}$ and $\mathrm{Yb}_{2} \mathrm{Sn}_{2} \mathrm{O}_{7}$. On the other hand, at extremely low fields it does $[13,18]$. Quantum perturbation theory describes $P_{Z}(t)$ in terms of correlation functions when the spin dynamics 
is sufficiently fast [30,47-49], namely $v_{\mathrm{c}} /\left(\gamma_{\mu} \Delta_{\mathrm{LF}}\right) \gtrsim 2$ [26]. It has been shown that this quantum description and the one exposed in Sec. II give consistent results in this limit when the field component distribution is Gaussian [26], as in the paramagnetic state of the two compounds of interest. Remarkably, at extremely low $B_{\text {ext }}$ the quantum description leads to a monotonic decay of $P_{Z}(t)$ which is exponential-like for $v_{\mathrm{c}} t \gtrsim 1[49,50]$. In our case, the shortest time we probed is $t \simeq 0.1 \mu \mathrm{s}$. This means that $v_{\mathrm{c}} \gtrsim 10 \mu \mathrm{s}^{-1}$, a lower bound larger than the value extracted from the analysis of $A^{\mathrm{LF}}(t)$ measured under field which is $v_{\mathrm{c}} \approx 1 \mu \mathrm{s}^{-1}$. Hence the fluctuation modes probed at extremely low field are fluctuating much faster than at intermediate field.

This discussion gives results entirely consistent with previous inferences from extremely low $B_{\text {ext }}$ measurements near $T_{\mathrm{c}}$ in the paramagnetic state $[13,18]$. From ${ }^{170} \mathrm{Yb}$ Mössbauer measurements the fluctuation rate for $\mathrm{Yb}_{2} \mathrm{Ti}_{2} \mathrm{O}_{7}$ and $\mathrm{Yb}_{2} \mathrm{Sn}_{2} \mathrm{O}_{7}$ was found to be in the $10^{10} \mathrm{~s}^{-1}$ range. Identifying $\mu \mathrm{SR}$ and Mössbauer fluctuation rates, taking into account the measured spin-lattice relaxation rate $\lambda_{Z}$ characterizing the decay of $P_{Z}(t)$, and the conventional motional narrowing formula $\lambda_{Z}=2 \gamma_{\mu}^{2} \Delta_{\mathrm{LF}}^{2} / v_{\mathrm{c}}, \Delta_{\mathrm{LF}}$ was computed to be consistent with expectation for the known $\mathrm{Yb}^{3+}$ magnetic moments.

At this juncture we note that the field standard deviation related to the slow dynamics is more than one order of magnitude smaller than the one characterizing the faster dynamics. This means that only a small fraction of the $\mathrm{Yb}^{3+}$ magnetic modes is involved in the slow dynamics.

Surprisingly, no spontaneous $\mu$ SR oscillation is observed below $T_{\mathrm{c}}$ for the stannate. Such a lack of oscillation was also observed for $\mathrm{Tb}_{2} \mathrm{Sn}_{2} \mathrm{O}_{7}$ and interpreted as a signature of the dynamical nature of the ground state of the compound [34], later on confirmed by neutron-spin echo measurements [51]. This means that $\tilde{v}_{\mathrm{c}}>2 \gamma_{\mu} B_{\text {fluc }}$, where $\tilde{v}_{\mathrm{c}}$ is the fluctuation rate of the fluctuating spontaneous field $B_{\text {fluc }}$ at the muon site [26]. From the relaxation rate $\lambda_{Z} \simeq 2.3 \mu \mathrm{s}^{-1}$ measured at low temperature in $\mathrm{Tb}_{2} \mathrm{Sn}_{2} \mathrm{O}_{7}$ (Ref. [34]) and from the fluctuation rate $\tilde{v}_{\mathrm{c}}=5 \times 10^{10} \mathrm{~s}^{-1}$ measured with the neutron spin echo technique, we deduce $B_{\text {fluc }}=0.4 \mathrm{~T}$ using the formula $\lambda_{Z}=$ $\gamma_{\mu}^{2} B_{\text {fluc }}^{2} / v_{\mathrm{c}}$. Scaling $B_{\text {fluc }}$ to the ordered magnetic moments$5.4 \mu_{\mathrm{B}}$ in the $\mathrm{Tb}_{2} \mathrm{Sn}_{2} \mathrm{O}_{7}$ and approximately $1.1 \mu_{\mathrm{B}}$ in the $\mathrm{Yb}$ pyrochlores $[13,18]$ —we compute $B_{\text {fluc }} \simeq(1.1 / 5.4) \times 0.4=$ $0.08 \mathrm{~T}$ for the two ytterbium compounds. Therefore, $\tilde{v}_{\mathrm{c}}>$ $7 \times 10^{7} \mathrm{~s}^{-1}$.

Hence the analysis of the $\mu$ SR data leads to two magnetic fluctuation rates in the paramagnetic state: $\approx 10^{6} \mathrm{~s}^{-1}$ and $\approx 10^{10} \mathrm{~s}^{-1}$ close to $T_{\mathrm{c}}$. In the ordered state two rates are also detected: again $\approx 10^{6} \mathrm{~s}^{-1}$ and a rate larger than $7 \times 10^{7} \mathrm{~s}^{-1}$. Finding more than one fluctuation mode is not surprising: we refer to the ordered state of $\mathrm{Tb}_{2} \mathrm{Sn}_{2} \mathrm{O}_{7}$ [34,51-53].

\section{CONCLUSIONS}

In this paper we have presented a complete $\mu \mathrm{SR}$ study of the spin dynamics of $\mathrm{Yb}_{2} \mathrm{Ti}_{2} \mathrm{O}_{7}$ and $\mathrm{Yb}_{2} \mathrm{Ti}_{2} \mathrm{O}_{7}$ as seen in a modest applied field. Extremely slow fluctuation modes with a scale of approximately $10^{6} \mathrm{~s}^{-1}$ were found both below and above the transition temperature. On the other hand, a much faster fluctuation rate, namely in the $10^{10} \mathrm{~s}^{-1}$ range near $T_{\mathrm{c}}$, was recognized from extremely low field paramagnetic measurements. In addition, a scale larger than $7 \times 10^{7} \mathrm{~s}^{-1}$ does exist in the ordered state. A similar spectrum of fluctuation rates is present in the two compounds. Such a broad and complex spectrum of fluctuations is unusual in a magnetic system. To our knowledge no theory is available for comparison.

The field component distribution deviates from the Gaussian shape for the two compounds below $T_{\mathrm{c}}$, pointing out the existence of short-range magnetic correlations. This is an unconventional feature which suggests the formation of molecular spin substructures, such as spin loops. They probably also exist in the paramagnetic state, although a signature of them has only been found from specific heat. Finally our study reveals that the magnetic susceptibility of $\mathrm{Yb}_{2} \mathrm{Ti}_{2} \mathrm{O}_{7}$ is much stronger than that of $\mathrm{Yb}_{2} \mathrm{Sn}_{2} \mathrm{O}_{7}$ below $T_{\mathrm{c}}$.

Still considering the ytterbium pyrochlore family, we note that ferromagnetic superexchange interactions drive the magnetic transition observed at $T_{\mathrm{c}}$ for $\mathrm{Yb}_{2} \mathrm{Ti}_{2} \mathrm{O}_{7}$ and $\mathrm{Yb}_{2} \mathrm{Sn}_{2} \mathrm{O}_{7}$. These compounds occur to be spin-ice like. It would be of interest to investigate with $\mu$ SR techniques $\mathrm{Yb}_{2} \mathrm{Ge}_{2} \mathrm{O}_{7}$ for which antiferromagnetic superexchange interactions are responsible for the magnetic transition [54]. In particular, possible differences in the spin dynamics would be of interest. In this respect, we note that the relaxation for the antiferromagnet $\mathrm{Er}_{2} \mathrm{Ti}_{2} \mathrm{O}_{7}$ is strikingly different from that observed in this paper $[55,56]$. In particular, the field responses below $T_{\mathrm{c}}$ are quite different.

\section{ACKNOWLEDGMENTS}

This research project has been partially supported by the European Commission under the 6th Framework Programme through the Key Action: Strengthening the European Research Area, Research Infrastructures (Contract No. RII3-CT-2003505925) and under the 7th Framework Programme through the 'Research Infrastructures' action of the 'Capacities' Programme, Contracts No. CP-CSA_INFRA-2008-1.1.1 and No. 226507-NMI3. The $\mu$ SR measurements were performed at $\mathrm{S} \mu \mathrm{S}$, Paul Scherrer Institute, Villigen, Switzerland, and at the ISIS facility, Rutherford Appleton Laboratory, Chilton, UK. A.Y. gratefully acknowledges partial support of Professor $\mathrm{H}$. Keller from the University of Zurich for the $\mu \mathrm{SR}$ measurements.
[1] M. J. Harris, S. T. Bramwell, D. F. McMorrow, T. Zeiske, and K. W. Godfrey, Phys. Rev. Lett. 79, 2554 (1997).

[2] T. Fennell, P. P. Deen, A. R. Wildes, K. Schmalzl, D. Prabhakaran, A. T. Boothroyd, R. J. Aldus, D. F. McMorrow, and S. T. Bramwell, Science 326, 415 (2009).

[3] I. A. Ryzhkin, JETP 101, 481 (2005).
[4] C. Castelnovo, R. Moessner, and S. L. Sondhi, Nature (London) 451, 42 (2008).

[5] D. J. P. Morris, D. A. Tennant, S. A. Grigera, B. Klemke, C. Castelnovo, R. Moessner, C. Czternasty, M. Meissner, K. C. Rule, J.-U. Hoffmann, K. Kiefer, S. Gerischer, D. Slobinsky, and R. S. Perry, Science 326, 411 (2009). 
[6] J. S. Gardner, M. J. P. Gingras, and J. E. Greedan, Rev. Mod. Phys. 82, 53 (2010).

[7] L. Yin, J. S. Xia, Y. Takano, N. S. Sullivan, Q. J. Li, and X. F. Sun, Phys. Rev. Lett. 110, 137201 (2013).

[8] P. Dalmas de Réotier, A. Yaouanc, A. Bertin, C. Marin, S. Vanishri, D. Sheptyakov, A. Cervellino, B. Roessli, and C. Baines, J. Phys.: Conf. Ser. 551, 012021 (2014).

[9] A. Yaouanc, P. Dalmas de Réotier, A. Bertin, C. Marin, E. Lhotel, A. Amato, and C. Baines, Phys. Rev. B 91, 104427 (2015).

[10] J. Villain, Z. Phys. B 33, 31 (1979).

[11] S.-H. Lee, C. Broholm, W. Ratcliff, G. Gasparovic, Q. Huang, T. H. Kim, and S.-W. Cheong, Nature (London) 418, 856 (2002).

[12] M. Hermele, M. P. A. Fisher, and L. Balents, Phys. Rev. B 69, 064404 (2004).

[13] J. A. Hodges, P. Bonville, A. Forget, A. Yaouanc, P. Dalmas de Réotier, G. André, M. Rams, K. Królas, C. Ritter, P. C. M. Gubbens, C. T. Kaiser, P. J. C. King, and C. Baines, Phys. Rev. Lett. 88, 077204 (2002).

[14] A. Yaouanc, P. Dalmas de Réotier, P. Bonville, J. A. Hodges, P. C. M. Gubbens, C. T. Kaiser, and S. Sakarya, Physica B 326, 456 (2003).

[15] J. S. Gardner, G. Ehlers, N. Rosov, R. W. Erwin, and C. Petrovic, Phys. Rev. B 70, 180404(R) (2004).

[16] Y. Yasui, M. Soda, S. Iikubo, M. Ito, M. Sato, N. Hamaguchi, T. Matsushita, N. Wada, T. Takeuchi, N. Aso, and K. Kakurai, J. Phys. Soc. Jpn. 72, 3014 (2003).

[17] L.-J. Chang, S. Onoda, Y. Su, Y.-J. Kao, K.-D. Tsuei, Y. Yasui, K. Kakurai, and M. R. Lees, Nat. Commun. 3, 992 (2012).

[18] A. Yaouanc, P. Dalmas de Réotier, P. Bonville, J. A. Hodges, V. Glazkov, L. Keller, V. Sikolenko, M. Bartkowiak, A. Amato, C. Baines, P. J. C. King, P. C. M. Gubbens, and A. Forget, Phys. Rev. Lett. 110, 127207 (2013).

[19] J. Lago, I. Živković, J. O. Piatek, P. Álvarez, D. Hüvonen, F. L. Pratt, M. Díaz, and T. Rojo, Phys. Rev. B 89, 024421 (2014).

[20] P. Dalmas de Réotier, V. Glazkov, C. Marin, A. Yaouanc, P. C. M. Gubbens, S. Sakarya, P. Bonville, A. Amato, C. Baines, and P. J. C. King, Physica B 374-375, 145 (2006).

[21] A. Yaouanc, P. Dalmas de Réotier, C. Marin, and V. Glazkov, Phys. Rev. B 84, 172408 (2011).

[22] A. Yaouanc, A. Maisuradze, and P. Dalmas de Réotier, Phys. Rev. B 87, 134405 (2013).

[23] L.-J. Chang, M. R. Lees, I. Watanabe, A. D. Hillier, Y. Yasui, and S. Onoda, Phys. Rev. B 89, 184416 (2014).

[24] R. M. D’Ortenzio, H. A. Dabkowska, S. R. Dunsiger, B. D. Gaulin, M. J. P. Gingras, T. Goko, J. B. Kycia, L. Liu, T. Medina, T. J. Munsie, D. Pomaranski, K. A. Ross, Y. J. Uemura, T. J. Williams, and G. M. Luke, Phys. Rev. B 88, 134428 (2013).

[25] E. Lhotel, S. R. Giblin, M. R. Lees, G. Balakrishnan, L. J. Chang, and Y. Yasui, Phys. Rev. B 89, 224419 (2014).

[26] A. Yaouanc and P. Dalmas de Réotier, Muon Spin Rotation, Relaxation, and Resonance: Applications to Condensed Matter (Oxford University Press, Oxford, 2011).

[27] In the longitudinal geometry, the beamline spin rotator is supplied with low voltage and current to act as a separator, namely a device used to remove unwanted particles, e.g., electrons, from the beam. See http://www.psi.ch/smus/pim3.

[28] In fact, at LTF the angle between $\mathbf{S}_{\mu}$ and $\mathbf{B}_{\text {ext }}$ is about $57^{\circ}$.

[29] K. W. Kehr, G. Honig, and D. Richter, Z. Phys. B 32, 49 (1978).

[30] R. S. Hayano, Y. J. Uemura, J. Imazato, N. Nishida, T. Yamazaki, and R. Kubo, Phys. Rev. B 20, 850 (1979).
[31] P. Dalmas de Réotier, A. Yaouanc, and A. Maisuradze, J. Phys.: Conf. Ser. 551, 012005 (2014).

[32] Note that, in addition, we expect the field component distribution measured on a powder to be symmetric, provided there is no field-induced anisotropy.

[33] D. R. Noakes, J. Phys.: Condens. Matter 11, 1589 (1999).

[34] P. Dalmas de Réotier, A. Yaouanc, L. Keller, A. Cervellino, B. Roessli, C. Baines, A. Forget, C. Vaju, P. C. M. Gubbens, A. Amato, and P. J. C. King, Phys. Rev. Lett. 96, 127202 (2006).

[35] B. G. Ueland, G. C. Lau, R. J. Cava, J. R. O'Brien, and P. Schiffer, Phys. Rev. Lett. 96, 027216 (2006).

[36] P. P. Deen, O. A. Petrenko, G. Balakrishnan, B. D. Rainford, C. Ritter, L. Capogna, H. Mutka, and T. Fennell, Phys. Rev. B 82, 174408 (2010).

[37] A. Bertin, P. Dalmas de Réotier, B. Fåk, C. Marin, A. Yaouanc, A. Forget, D. Sheptyakov, B. Frick, C. Ritter, A. Amato, C. Baines, and P. J. C. King (unpublished).

[38] M. Taillefumier, J. Robert, C. L. Henley, R. Moessner, and B. Canals, Phys. Rev. B 90, 064419 (2014).

[39] J. P. C. Ruff, J. P. Clancy, A. Bourque, M. A. White, M. Ramazanoglu, J. S. Gardner, Y. Qiu, J. R. D. Copley, M. B. Johnson, H. A. Dabkowska, and B. D. Gaulin, Phys. Rev. Lett. 101, 147205 (2008).

[40] H. W. J. Blöte, R. F. Wielinga, and W. J. Huiskamp, Physica 43, 549 (1969).

[41] P. Dalmas de Réotier, A. Yaouanc, P. C. M. Gubbens, C. T. Kaiser, C. Baines, and P. J. C. King, Phys. Rev. Lett. 91, 167201 (2003).

[42] P. Bonville, J. A. Hodges, E. Bertin, J. P. Bouchaud, M. Ocio, P. Dalmas de Réotier, L. P. Regnault, H. M. Ronnow, J. P. Sanchez, S. Sosin, A. Yaouanc, M. Rams, and K. Krolas, in Proceedings of the XXXVIII Zakopane School of Physics "Condensed matter studies with nuclear methods,” Zakopane (Poland), edited by E. A. Görlich, K. Królas, and A. T. Pedziwiatr (Krakow, 2003).

[43] P. Bonville, J. A. Hodges, E. Bertin, J.-P. Bouchaud, P. Dalmas de Réotier, L.-P. Regnault, H. M. Rønnow, J.-P. Sanchez, S. Sosin, and A. Yaouanc, Hyperfine Interact. 156-157, 103 (2004).

[44] Y. J. Uemura, A. Keren, K. Kojima, L. P. Le, G. M. Luke, W. D. Wu, Y. Ajiro, T. Asano, Y. Kuriyama, M. Mekata, H. Kikuchi, and K. Kakurai, Phys. Rev. Lett. 73, 3306 (1994).

[45] A. Yaouanc, P. Dalmas de Réotier, Y. Chapuis, C. Marin, S. Vanishri, D. Aoki, B. Fåk, L. P. Regnault, C. Buisson, A. Amato, C. Baines, and A. D. Hillier, Phys. Rev. B 84, 184403 (2011).

[46] A. Amato, P. Dalmas de Réotier, D. Andreica, A. Yaouanc, A. Suter, G. Lapertot, I. M. Pop, E. Morenzoni, P. Bonfà, F. Bernardini, and R. De Renzi, Phys. Rev. B 89, 184425 (2014).

[47] V. G. Baryshevskiı̌ and S. A. Kuten', Fiz. Tverd. Tela (Leningrad) 18, 2873 (1976) [Sov. Phys. Solid State 18, 1677 (1976)].

[48] T. McMullen and E. Zaremba, Phys. Rev. B 18, 3026 (1978).

[49] P. Dalmas de Réotier and A. Yaouanc, J. Phys.: Condens. Matter 4, 4533 (1992).

[50] A. Keren, Phys. Rev. B 50, 10039 (1994).

[51] K. C. Rule, G. Ehlers, J. S. Gardner, Y. Qiu, E. Moskvin, K. Kiefer, and S. Gerischer, J. Phys.: Condens. Matter 21, 486005 (2009).

[52] Y. Chapuis, A. Yaouanc, P. Dalmas de Réotier, S. Pouget, P. Fouquet, A. Cervellino, and A. Forget, J. Phys.: Condens. Matter 19, 446206 (2007). 
[53] I. Mirebeau, H. Mutka, P. Bonville, A. Apetrei, and A. Forget, Phys. Rev. B 78, 174416 (2008).

[54] Z. L. Dun, M. Lee, E. S. Choi, A. M. Hallas, C. R. Wiebe, J. S. Gardner, E. Arrighi, R. S. Freitas, A. M. Arevalo-Lopez, J. P. Attfield, H. D. Zhou, and J. G. Cheng, Phys. Rev. B 89, 064401 (2014).
[55] J. Lago, T. Lancaster, S. J. Blundell, S. T. Bramwell, F. L. Pratt, M. Shirai, and C. Baines, J. Phys.: Condens. Matter 17, 979 (2005).

[56] P. Dalmas de Réotier, A. Yaouanc, Y. Chapuis, S. H. Curnoe, B. Grenier, E. Ressouche, C. Marin, J. Lago, C. Baines, and S. R. Giblin, Phys. Rev. B 86, 104424 (2012). 